\section{Ataxia espinocerebelosa tipo 3 (enfermedad de Machado-Joseph) y vareniclina}

\section{Spinocerebellar ataxia type 3 (Machado-Joseph disease) and varenicline}

\section{Sr. Editor:}

Hemos leído con atención la carta del Dr. Marcelo Miranda, publicada en la Revista Médica de Chile, donde menciona que "lamentablemente, los ensayos terapéuticos han sido infructuosos en modificar el curso de la enfermedad y el manejo sigue siendo sintomático". Queremos relatar nuestra experiencia en una paciente con ataxia espino cerebelosa tipo 3 (AET3) (enfermedad de Machado-Joseph) medicada con vareniclina:

Mujer de 53 años sin antecedentes propios ni familiares de importancia, que comenzó hace 19 años con inestabilidad en la marcha y hace 9 años se agregó disartria. Fue examinada por nosotros hace 3 años, mostrando en el examen neurológico un nistagmus horizontal bidireccional, diplopía horizontal intermitente, prueba índice nariz y talón rodilla con dismetría bilateral, marcha atáxica y signo de Romberg positivo. La analítica completa fue normal. La Resonancia Magnética de cerebro fue normal, sin observarse atrofia cerebelosa. El estudio de la mutación mostró 68 repeticiones de la secuencia CAG (citosina-adenina-guanina) del gen ATXN3 lo que confirmó esta entidad (Laboratorio de Neurogenética, Londres, Reino Unido). Se inició tratamiento con vareniclina (con el mismo esquema usado para la cesación tabáquica) llegando a una dosis de un miligramo cada $12 \mathrm{~h}^{2}$. La paciente presentó una mejoría inicial (de la coordinación, balance y marcha) a los 10 días, sosteniéndola objetivamente durante aproximadamente un año, después de lo cual se perdió dicho efecto.

Varios estudios recientes indican que la vareniclina, un agonista parcial del receptor nicotínico $\alpha 4 \beta 2$, mejora los síntomas de pacientes con ataxia neurodegenerativa de distintas etiologías ${ }^{2,3}$. Los mecanismos de acción propuestos en base a estudios animales son múltiples: incremento de sobrevida y conectividad de neuronas olivocerebelosas; reducción de las disquinesias producidas por L-dopa; liberación de dopamina a nivel presináptico; incremento de la activación funcional del vermis cerebeloso e incremento del flujo cerebral del circuito tálamo-cortical y protección contra la muerte de neuronas motoras en cultivos de medula espinal ${ }^{2,3}$. Todo ello sería por la presencia de múltiples receptores nicotínicos en el cerebelo (ubicados en la células granulares, células de Purkinje e interneuronas), además del resto del cerebro, en orden de concentración decreciente $(\alpha 4 \beta 2>\alpha 3 \beta 4>\alpha 3 \beta 2 \beta 4>\alpha 3 \alpha 4 \beta 2)^{2,3}$. Los efectos clínicos de los agonistas nicotínicos fueron reproducidos en modelos animales de ataxia olivocerebelosa ${ }^{3}$. A pesar de las críticas metodológicas al trabajo de Zesiewicz et al, el último consenso de la Sociedad Europea de Neurología de diagnóstico y tratamiento de ataxias crónicas del adulto incluye a la vareniclina para el tratamiento de la AET3, con nivel B de evidencia ${ }^{2,4-6}$.

Nuevos hallazgos han señalado que la mutación del gen ATXN3 (ubicado en el brazo largo del cromosoma 14) produce un incremento de las expansiones CAG, induciendo acumulación de residuos largos de glutamina o poliglutamina (y toxicidad por proteínas), lo que resulta en la enfermedad $^{7}$. Además, se observó que las repeticiones de la secuencia CAG del gen ATXN3 inhiben la actividad de la polinucleótido quinasa 3 fosfatasa (PNKP) con la consiguiente acumulación de ADN dañado ${ }^{8}$. Se ha observado una disminución de la actividad enzimática en modelos animales sobre las regiones de sistema nervioso afectadas por la AET3 y en cerebros de pacientes con AET3, dado que existe una acumulación significativa del ADN dañado. Se ha demostrado que la acumulación del ADN dañado indujo muerte neuronal, lo que es un hallazgo común en estos pacientes. Será importante determinar si la normalización de los niveles y/o actividad de ésta enzima beneficiaría a estos pacientes.

Mendonça et al han realizado un trasplante de células madre en el cerebelo de ratones adultos con AET3, observando que estas células se diferenciaron en neuronas, astrocitos y oligodendrocitos, lo que redujo significativamente los síntomas motores de estos animales. Además observaron una disminución de la pérdida de las células de Purkinje, reducción de la inflamación e incremento de factores neurotróficos ${ }^{9}$. El factor de crecimiento nervioso (FCN) forma parte de la familia de las neurotrofinas y es esencial para el desarrollo, diferenciación y supervivencia del cerebro de los mamíferos. Un estudio prospectivo no aleatorizado que incluyó 21 pacientes con AET3 a quienes se les administró FCN mostró 
mejoría de los síntomas a las dos semanas de finalizado el tratamiento y que se mantuvo luego de las cuatro semanas ${ }^{10}$. Se postularon dos posibles mecanismos: que la aplicación periférica de FCN tuvo efectos directos sobre el cerebelo ya que el FCN y su receptor de alta afinidad, que pertenece a la familia tirosina quinasa, se encuentran en neuronas cerebelosas $y$, alternativamente, el efecto del FCN podría ser mediado a través del sistema propioceptivo que también se compromete en esta enfermedad.

Muchos esfuerzos serán necesarios para que estas estrategias puedan ser usadas clínicamente.

Agradecimientos: Al Dr. Henry Houlden, del University College London, Institute of Neurology, quien realizó el estudio genético de la paciente.

Pablo Young , Bárbara C. Finn ${ }^{1}$, Franco Giuliani ${ }^{1}$, Ricardo Reisin ${ }^{2}$ ${ }^{1}$ Servicio de Clínica Médica.

${ }^{2}$ Servicio de Neurología, Hospital Británico de Buenos Aires, Argentina.

\section{Referencias}

1. Miranda MC. Diagnóstico de Ataxia Espinocerebelosa tipo 3 (Enfermedad de Machado-Joseph) en Chile. Rev Med Chile 2015; 143: 126-7.

2. Zesiewicz TA, Greenstein PE, Sullivan KL, Wecker L, Miller A, Jahan I, et al. A randomized trial of varenicline (Chantix) for the treatment of spinocerebellar ataxia type 3. Neurology 2012; 78: 545-50.

3. Wecker L, Engberg ME, Philpot RM, Lambert CS, Kang CW, Antilla JC, et al. Neuronal nicotinic receptor agonists improve gait and balance in olivocerebellar ataxia. Neuropharmacology 2013; 73: 75-86.

4. Filla A, Sacca F, De Michele G. A randomized trial of varenicline (Chantix) for the treatment of spinocerebellar ataxia type 3. Neurology 2012; 78: 1538.
5. Connolly BS, Prashanth LK, Shah BB, Marras C, Lang AE. A randomized trial of varenicline (chantix) for the treatment of spinocerebellar ataxia type 3. Neurology 2012; 79: 2218.

6. van de Warrenburg BP, van Gaalen J, Boesch S, Burgunder JM, Dürr A, Giunti P, et al. EFNS/ENS Consensus on the diagnosis and management of chronic ataxias in adulthood. Eur J Neurol 2014; 21: 552-62.

7. Ramani B, Harris GM, Huang R, Seki T, Murphy GG, Costa Mdo C, et al. A knockin mouse model of spinocerebellar ataxia type 3 exhibits prominent aggregate pathology and aberrant splicing of the disease gene transcript. Hum Mol Genet 2015; 24: 1211-24.

8. Chatterjee A, Saha S, Chakraborty A, Silva-Fernandes A, Mandal SM, Neves-Carvalho A, et al. The Role of the Mammalian DNA End-processing Enzyme Polynucleotide Kinase 3'-Phosphatase in SpinocerebellarAtaxia Type 3 Pathogenesis. PLoSGenet 2015; 11: e1004749.

9. Mendonça LS, Nóbrega C, Hirai H, Kaspar BK, Pereira de Almeida L.Transplantation of cerebellar neural stem cells improves motor coordination and neuropathology in Machado-Joseph disease mice. Brain 2015; 138: 32035.

10. Tan S, Wang RH, Niu HX, Shi CH, Mao CY, Zhang R, et al. Nerve Growth Factor for the Treatment of Spinocerebellar Ataxia Type 3: An Open-label Study. Chin Med J (Engl)2015; 128: 291-4.

Nota del Editor: La breve mejoría experimentada por la paciente referida en esta carta apoya la conclusión expresada en la Referencia 1: "lamentablemente, los ensayos terapéuticos han sido infructuosos en modificar el curso de la enfermedad y el manejo sigue siendo sintomático".

Correspondencia a:

Dr. Pablo Young

Hospital Británico. Perdriel 74 (1280) Buenos Aires, Argentina. Tel 541143096400 pabloyoung2003@yahoo.com.ar 